\title{
Chemical Assembly of Copper Oxide and Single Walled Carbon Nanotubes for Enhanced Photocatalytic Dye Degradation under Solar Light Irradiation ${ }^{\dagger}$
}

\author{
Kamal Prasad Sapkota ${ }^{1,2}$, Md. Akherul Islam ${ }^{1}$, Md. Abu Hanif ${ }^{1}$, Jeasmin Akter ${ }^{1}$ and Jae Ryang Hahn ${ }^{1,3, *}$ \\ 1 Department of Chemistry and Department of Bioactive Material Sciences, Research Institute of Physics and \\ Chemistry, Jeonbuk National University, Jeonju 54896, Korea; mychemistry2037@gmail.com (K.P.S.); \\ akherulraju@gmail.com (M.A.I.); hanif4572@gmail.com (M.A.H.); tina44445@gmail.com (J.A.) \\ 2 Department of Chemistry, Amrit Campus, Tribhuvan University, Kathmandu 44618, Nepal \\ 3 Textile Engineering, Chemistry and Science, North Carolina State University, 2401 Research Drive, \\ Raleigh, NC 27695-8301, USA \\ * Correspondence: jrhahn@jbnu.ac.kr \\ + Presented at the 2nd International Online-Conference on Nanomaterials, 15-30 November 2020; Available \\ online: https://iocn2020.sciforum.net/.
}

Citation: Sapkota, K.P.; Islam, M.A.; Hanif, M.A.; Akter, J.; Hahn, J.R. Chemical Assembly of Copper Oxide and Single Walled Carbon Nanotubes for Enhanced Photocatalytic Dye Degradation under Solar Light Irradiation. Mater. Proc. 2021, 4, 18. https://doi.org/ 10.3390/IOCN2020-07790

Academic Editors: Ana María Díez-Pascual, Antonio Di Bartolomeo and Guanying Chen

Published: 10 November 2020

Publisher's Note: MDPI stays neutral with regard to jurisdictional claims in published maps and institutional affiliations.

Copyright: $(\odot) 2020$ by the authors. Licensee MDPI, Basel, Switzerland. This article is an open access article distributed under the terms and conditions of the Creative Commons Attribution (CC BY) license (http://creativecommons.org/licenses/by/4.0/).

\begin{abstract}
We elaborate the synthesis and remarkable photocatalytic efficiency of a series of heterojunction nanocomposites with a cauliflower-like architecture composed of copper oxide $(\mathrm{CuO})$ and single-walled carbon nanotubes (SWCNTs). The photocatalysts with such a peculiar design were constructed via facile recrystallization followed by calcination and were symbolized as $\mathrm{CuO}$ SWCNT-1, CuO-SWCNT-2, and CuO-SWCNT-3, representing the components and calcination time in hours. The photocatalytic efficiency of the synthesized nanocomposite samples were investigated by evaluating the decomposition of methylene blue (MB) solution under natural sunlight exposure. All of the as-synthesized photocatalysts were substantially effectual for the photo-deterioration of MB solution. Moreover, CuO-SWCNT-3 revealed the top photocatalytic capability with 96\% decomposition of MB solution in $2 \mathrm{~h}$ while being exposed to visible light. Pristine $\mathrm{CuO}$ nanocrystals and the SWCNTs were employed as controls, whereas the photocatalytic performance of the heterocomposites was significantly better than that of pure $\mathrm{CuO}$ as well as SWCNTs. The recyclability of the photocatalysts was also explored, and the results asserted that the samples could be reused for five cycles without being altered notably in photocatalytic performance or morphology.
\end{abstract}

Keywords: CuO-SWCNT; nano-cauliflower; methylene blue; recrystallization; photo-deterioration; nanocomposites; photocatalysis

\section{Introduction}

Persistent organic pollutants impart adverse effects on human health via various routes, including endocrine disorders, congenital defects, organ malfunction, chronic illness and even death in some cases [1-3]. The degradation of such pollutants through photocatalytic reactions are being explored extensively by the researchers because of their capability to generate robust oxidizing radicals under solar irradiation that mineralize persistent organic pollutants completely through oxidation reactions [4]. Nanoscale semiconductor metal oxides, such as copper oxide $(\mathrm{CuO})$, and their composites, are among the popular agents for efficient photocatalytic activity [5]. $\mathrm{CuO}$ is a narrow bandgap semiconductor with plentiful availability, non-toxicity, low cost, p-type semi-conductivity, excellent catalytic proficiency, and excellent chemical stability. Nevertheless, there are some limitations in the practical use of $\mathrm{CuO}$ as an efficient photocatalyst. Comparatively feebler visible light absorption, altered accessibility to reaction sites, and the higher likelihood of 
the recombination of photon-generated electron-hole pairs come up as the central issues [6-8].

We improved the limitations of $\mathrm{CuO}$ via its chemical combination with the walls of SWCNTs through a straightforward recrystallization method accompanied by calcination. We prepared a group of CuO-SWCNT hetero-composites maintaining the recrystallization parameters constant and changing the durations of calcinations at a preset temperature $\left(550{ }^{\circ} \mathrm{C}\right)$. We named the as-prepared composites as CuO-SWCNT-1, CuOSWCNT-2, and CuO-SWCNT-3. We used those nanocomposites for the photocatalytic decomposition of methylene blue solution in natural sunlight exposure to convert it into harmless end products. The recycling potentiality of the as-synthesized composites was evaluated through their photocatalytic actions for five consecutive cycles. We found no substantial decrease in the photocatalytic activity and changes in their surface features after their recycling.

\section{Materials and Methods}

A homogeneous solution of copper (II) acetate hydrate $(6.0 \mathrm{~g})$ was prepared by dissolving it in ethanol $(100 \mathrm{~mL})$ in a beaker using bath sonication for an hour at room temperature $\left(20^{\circ} \mathrm{C}\right)$. SWCNTs $(100 \mathrm{mg})$ were released into the above solution, and the resulting mixture was devised for magnetic stirring for one more hour. The magnetic stirring bar was withdrawn and the mixture was left untouched for recrystallization for $8 \mathrm{~h}$. Recrystallized form of copper (II) acetate crystals-SWCNT aggregation was isolated from ethanol through vacuum filtration and the residual mass was dried at $70{ }^{\circ} \mathrm{C}$ in a previously heated oven for $2 \mathrm{~h}$ so that it is completely free of ethanol. The dried mass was finally calcined in a muffle furnace at $550{ }^{\circ} \mathrm{C}$ to construct $\mathrm{CuO}-\mathrm{SWCNT}$ heterostructures. The detailed method of synthesis of nanocomposite catalysts is shown by a flowchart (Fig. $1)$.

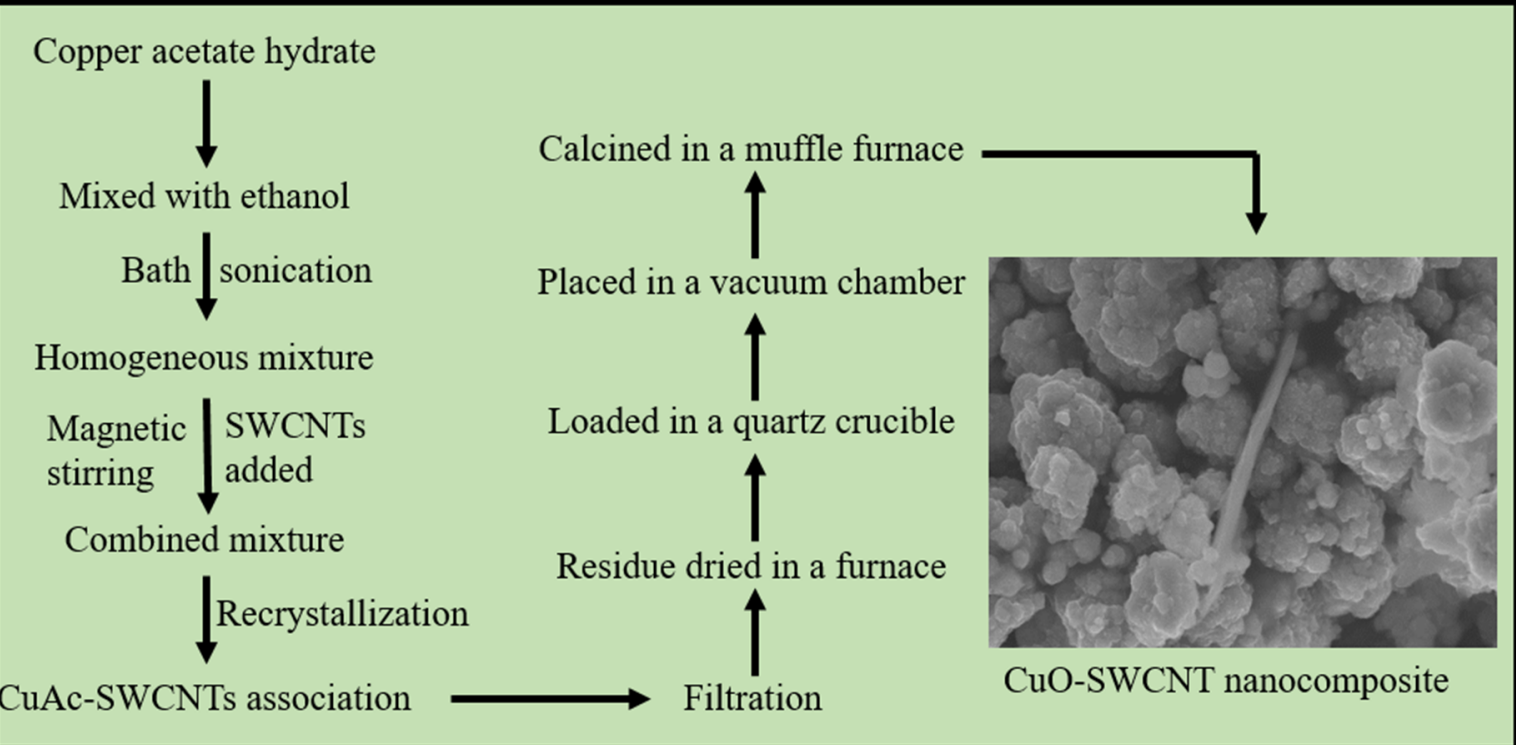

Figure 1. A flowchart depicting the method of synthesis of CuO-SWCNT nanocomposites.

\section{Results}

$\mathrm{CuO}$ nanocrystals chemically combined with the outer walls of SWCNTS and have resulted in the generation of the cauliflower-shaped nanocomposite particles with various sizes that range between $30 \mathrm{~nm}$ to $1 \mu \mathrm{m}$. Figure $2 \mathrm{a}$, b demonstrate the representative FE SEM images of the as-synthesized composites in the nano-cauliflower shape. Figure 2c, $d$ demonstrate the HR-TEM portrayals of the composites in which the chemical attachment 
of the constituents is evident. However, the nano-cauliflower architecture has been demolished in TEM images due to the strong bath sonication while preparing TEM samples. The size of the CuO-nanocrystals surrounding the SWCNTs, as measured during HRTEM analysis, ranges between 2 to $15 \mathrm{~nm}$.

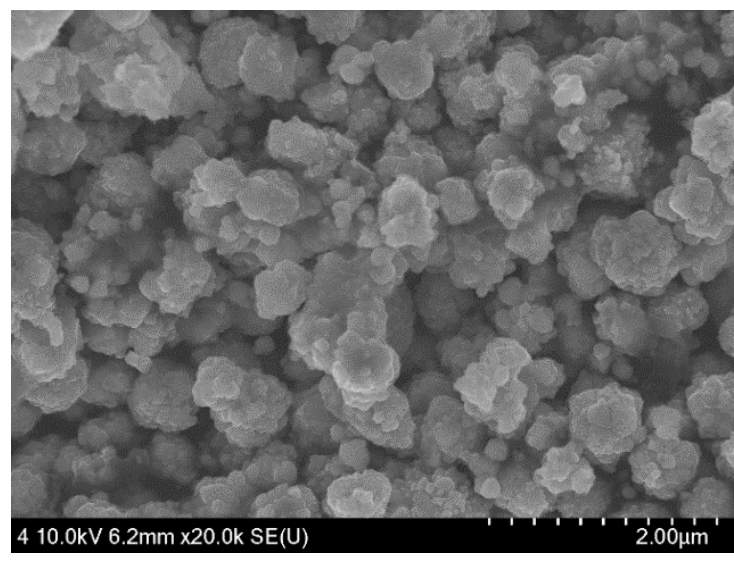

(a)

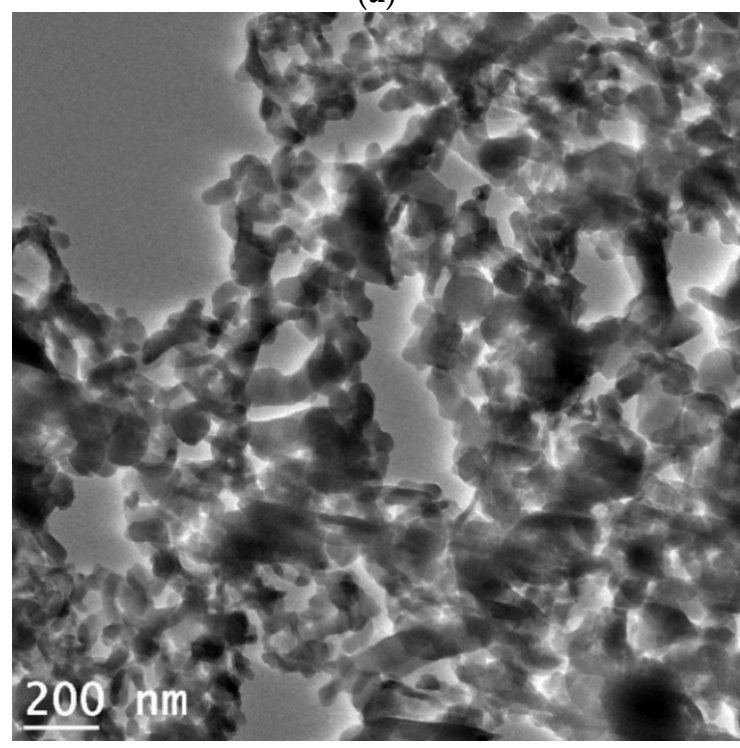

(c)

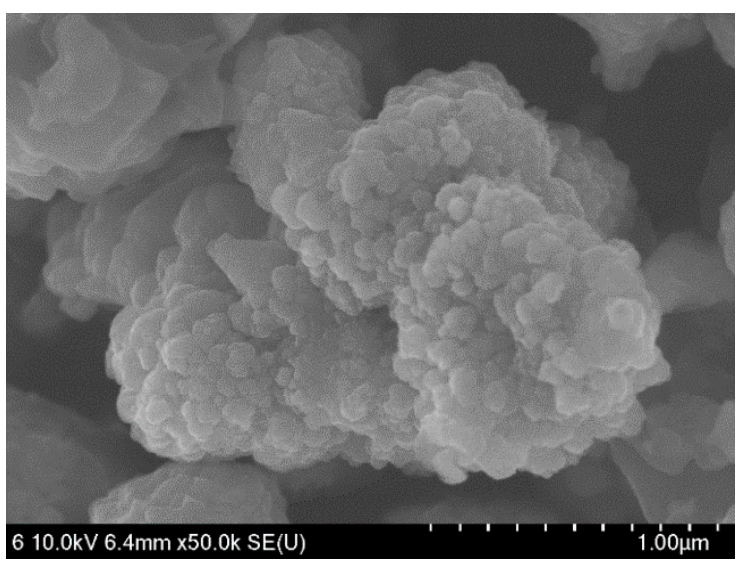

(b)

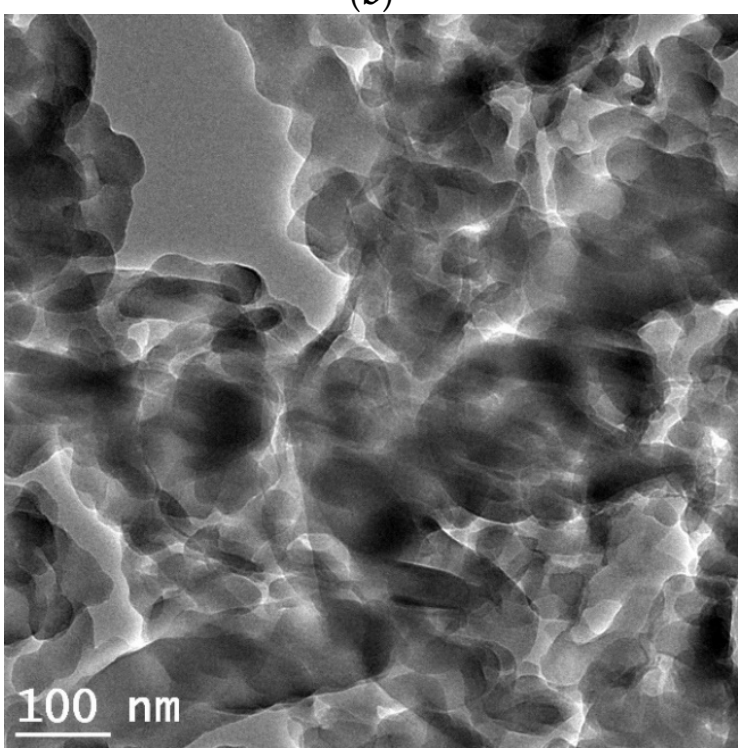

(d)

Figure 2. Representative FE-SEM images displaying a cauliflower-like appearance $(\mathbf{a}, \mathbf{b})$ and HR-TEM micrographs (c, d) of the CuO-SWCNTs heterostructures.

X-ray diffraction (XRD) spectra of the samples confirmed monoclinic CuO geometry in the composite and no change in the crystal structure of SWCNTs during chemical combination. The crystallite size, which was calculated from the XRD profile using the Scherrer Equation, was found to be $23.95 \mathrm{~nm}$. The chemical bonding between $\mathrm{CuO}$ and SWCNTs was further confirmed by HR-XPS, which demonstrates the oxidation states and chemical environments of chemically combined $\mathrm{Cu}, \mathrm{O}$ and $\mathrm{C}$ in the nanocomposites. XPS results disclose that crystalline $\mathrm{CuO}$ and SWCNTs have combined through the origination of covalent bonds as $\mathrm{CuO}-\mathrm{SWCNT}$ or $\mathrm{Cu}-\mathrm{OOC}-\mathrm{SWCNT}$. As demonstrated via the XPS results, the TGA profiles further verified the chemical bonding between the SWCNTs and $\mathrm{CuO}$ nanocrystals. Such a chemical bond generates a permanent heterojunction between the surfaces of the constituents, which is crucial for the enhanced photocatalytic performance.

The improvement in the optical characteristics of the as-synthesized heterostructures were asserted through UV-vis diffuse reflectance spectra. Those spectra revealed a significant shrinkage in the bandgap energy of the composite samples compared to the pristine 
$\mathrm{CuO}$, affirming that chemical bonding with SWCNTs has improved the catalytic properties of the samples. Specific surface area, pore-size distribution and pore volume of the samples were examined via Brunauer-Emmett-Teller (BET) and Barrett-Joyner-Halenda $(\mathrm{BJH})$ analyses, and the findings display that the specific surface areas of the composite samples have augmented compared to pristine $\mathrm{CuO}$ due to its chemical bonding with SWCNTs.

The as-prepared $\mathrm{CuO}-\mathrm{SWCNT}$ photocatalysts were investigated for their photocatalytic efficiency by accessing the catalyzed break-down of the MB solution under natural sunlight exposure. Among the different samples, CuO-SWCNT-3 displayed the best photocatalytic performance achieving $96 \%$ photo-decomposition of MB solution through $2 \mathrm{~h}$ of natural sunlight irradiation. However, CuO-SWCNT-2 and CuO-SWCNT-1 caused $93 \%$ and $90 \%$ photo-degradation of the MB solution, respectively under similar conditions of exposure and at the same duration. The enhanced photocatalytic performance of the composites is credited to the increased visible light absorption by the photocatalysts, production of light-induced electron-hole sets and their separation to avoid recombination so that the charge carriers can participate in the redox reactions for the decomposition of organic dyes such as MB.

\section{Conclusions}

Our as-synthesized photocatalysts with interesting nano-cauliflower-like structures are capable of remarkable degradation of organic dyes such as MB solutions under natural sunlight exposure. The origination of heterojunctions between $\mathrm{CuO}$ and SWCNTs was confirmed via different characterization techniques such as HR-TEM, XRD, TGA/DSC and XPS. Among the different samples, CuO-SWCNT-3 displayed the best photocatalytic performance, achieving $96 \%$ photo-decomposition of MB solution in $2 \mathrm{~h}$ natural sunlight irradiation. Similarly, CuO-SWCNT-2 and CuO-SWCNT-1 caused 93\% and 90\% photo-degradation of the $\mathrm{MB}$ solution, respectively, under similar conditions of exposure and at the same duration. The results reveal that CuO-SWCNT nanocomposites can be used as efficient photocatalysts for the deterioration of water pollutants like organic dyes.

Author Contributions: J.R.H. supervised and provided resources, support and guidance in whole research work, K.P.S. designed and performed the experiments, analyzed data and wrote the article, A.I., A.H., J.A. analyzed data and read the manuscript rigorously to upgrade it.

Acknowledgments: This work was supported by Korean Government, NRF-2018R1A2B6006155.

Conflicts of Interest: The authors declare no conflict of interest.

\section{References}

1. Lyu, H.; Gao, B.; He, F.; Zimmerman, A.R.; Ding, C.; Tang, J.; Crittenden, J.C. Experimental and modeling investigations of ballmilled biochar for the removal of aqueous methylene blue. Chem. Eng. J. 2018, 335, 110-119.

2. Sapkota, K.P.; Lee, I.; Hanif, M.; Islam, M.; Hahn, J.R. Solar-light-driven efficient ZnO-single-walled carbon nanotube photocatalyst for the degradation of a persistent water pollutant organic dye. Catalysts 2019, 9, 498.

3. Othman, N.H.; Alias, N.H.; Shahruddin, M.Z.; Bakar, N.F.A.; Him, N.R.N.; Lau, W.J. Adsorption kinetics of methylene blue dyes onto magnetic graphene oxide. J. Environ. Chem. Eng. 2018, 6, 2803-2811.

4. Sapkota, K.P.; Lee, I.; Hanif, M.A.; Islam, M.A.; Akter, J.; Hahn, J.R. Enhanced Visible-Light Photocatalysis of Nanocomposites of Copper Oxide and Single-Walled Carbon Nanotubes for the Degradation of Methylene Blue. Catalysts 2020, $10,297$.

5. Li, H.; Su, Z.; Hu, S.; Yan, Y. Free-standing and flexible $\mathrm{Cu} / \mathrm{Cu} 2 \mathrm{O} / \mathrm{CuO}$ heterojunction net: A novel material as cost-effective and easily recycled visible-light photocatalyst. Appl. Catal. B Environ. 2017, 207, 134-142.

6. Hanif, M.; Lee, I.; Akter, J.; Islam, M.; Zahid, A.A.; Sapkota, K.P.; Hahn, J.R. Enhanced Photocatalytic and Antibacterial Performance of ZnO Nanoparticles Prepared by an Efficient Thermolysis Method. Catalysts 2019, 9, 608.

7. Kim, G.H.; Jeong, S.; Lee, I.; Hanif, M.A.; Islam, M.A.; Sapkota, K.P.; Hahn, J.R. Role of Electronic Structures and Dispersion Interactions in Adsorption Selectivity of Pyrimidine Molecules with a Si (5 5 12) Surface. J. Phys. Chem. C 2019, 123, $19506-19512$.

8. Sapkota, K.P.; Hassan, M.M.; Shrestha, S.; Hanif, M.A.; Islam, M.A.; Akter, J.; Abbas, H.G.; Hahn, J.R. Heterojunction formation between copper (II) oxide nanoparticles and single-walled carbon nanotubes to enhance antibacterial performance. Int. J. Pharm. 2020, 590, 119937. 